\title{
Performance Assessment of MC Placement for Multicast Routing in WDM Networks with Spare Light Splitting
}

\author{
I-Shyan Hwang, Tsung-Ching Lin and Zen-Der Shyu \\ Department of Computer Engineering and Science, \\ Yuan-Ze University, Chung-Li, Taiwan, 32026
}

\begin{abstract}
This investigation examines all-optical multicast routing in wavelength-routed optical networks with sparse Multicast Capable (MC) nodes that have three phases. The first phase is the MC node placement, and the maximum path count first (MPCF) algorithm is utilized to obtain candidates for MC nodes. The second phase is a multicast routing with MC-based schemes which including two algorithms, namely, the Pre-computing Minimum Cost Tree (PMC) and Pre-computing Shortest Path Tree (PSP) algorithms. Both algorithms use Steiner tree and shortest path tree to create an MC-based tree. The third phase is the system maintenance. System performance of PMC and PSP is compared with that of the Route to Source (RTS) algorithm in terms of average number of wavelengths needed - average call blocking probability and mean maximum transmission delay. Simulation results indicate that the proposed schemes utilize the minimum number of wavelengths needed and reduce blocking probability.
\end{abstract}

Keywords: Multicast Capable (MC), Light-tree, Maximum path count first (MPCF), Call blocking probability.

\section{Introduction}

As Internet traffic continues to increase exponentially, a wavelength division multiplexing (WDM) network with terabits per second bandwidth per fiber is a natural choice for the backbone of the next-generation optical Internet. Given that multicast is becoming increasingly, issues concerning the support for multicasting in Internet Protocol (IP) over WDM networks require investigation. To support multicast at the WDM layer, [1] introduced the concept of the light-tree, which is a point-to-multipoint extension of a light path. The primary benefit of light-trees is that only one transmitter is required for transmission and multiple destinations can share intermediate tree links. To support all-optical multicasting efficiently, nodes in a WDM network must have light splitting capability. A Multicast Capable (MC) node with splitting capability can forward an incoming message through multiple output channels. Implementing an MC node, however, is expensive. The concept of sparse-splitting was first introduced in [2]. With sparse-splitting, only a small number of nodes in the network are MCs, and the remainders are Multicast Incapable (MI). An MI node can forward an input signal to only one of the numerous output ports; thus, an MI node cannot serve as a branching node of a light-tree.

Route optimization in sparse-splitting networks is discussed in [2-5]. In [2], four heuristics, namely, Re-route-to-Source, Re-route-to-Any, Member-First, and Member-Only are proposed for generating multiple light-trees (i.e., a light forest) for a given multicast session, and thereby applying multiple transmitters and multiple wavelengths per session. In [3], a solution that generates a light-tree using single transmitter and single wavelength per session is presented. In [4], an all-optical multicast routing heuristic called $\mathrm{AOMH}$ is proposed that generates a light-tree with a single transmitter and multiple wavelengths per session. A virtual source-based multicast routing scheme is proposed in [5]. This mechanism creates mutual connectivity among all MC nodes in advance. In [6], that MI nodes are equipped with Tap-and-Continue ( $\mathrm{TaC})$ cross-connects is assumed. The cross-connect allows optical power to be harnessed while forwarding data to an output.

Existing multicast solutions for sparse-splitting WDM networks can be classified into source-based trees [2-3] and MC-based trees [4-5]. Source-based multicast schemes build light-trees in a way similar to traditional IP multicast trees, with routing algorithm modifications for adapting to the sparse-splitting constraint. In MC-based schemes, a light-tree connecting the $\mathrm{MC}$ nodes of a multicast group, called $M C$-tree, is first generated. Each MI node of the group then independently joins the nearest MC node that is already on the tree. Moreover, compared to source-based tree approaches, MC-based techniques have superior resource utilization and support for 
dynamic membership, shorter tree setup latency, and are more scalable to large networks.

Minimizing the number of wavelength channels are used for a multicast session is important because the number of wavelengths supported in a fiber link is limited. In this work, we will focus on developing an all-optical multicast routing algorithm that minimizes the number of wavelength channels required by a given multicast session. A light tree is built with minimum cost for a given multicast session. The cost of a light-tree is defined as the total number of wavelength channels utilized by a light-tree. In MC-based multicast routing methods, the cost of a resulting light-tree is dominated by the cost of the corresponding MC-tree because such a cost equals the cost of the MC-tree plus that of each light-path from an MI node to the MC-tree. Additionally, the cost of $\mathrm{MI}$ nodes connecting to the MC-tree also depends on the location of the nearest MC nodes of the MC-tree. Thus, finding the minimum-cost MC-tree is crucial to minimizing the total cost of the resulting light-tree.

\section{Problems Formulation}

The sparse-splitting optical network can be modeled as a directly connected graph $G(E, V)$, where $V$ and $E$ are the sets of nodes and directed links of the network, respectively. $\wedge(e)$ denote the set of wavelengths supported on link $e$ in the network. Given network $G$, $\wedge(e)$ for every $e \in E$, and a multicast session request $r(s, D)$, finding a multicast tree such that tree cost is minimized can be proved to be NP-hard. This is because minimizing tree cost can be regarded as minimizes the total number of wavelength channels utilized on the tree. In other words, this problem is equivalent to developing a multicast routing algorithm that minimizes total wavelength channel cost under a constraint of sparse light-splitting capability. Finding multicast routing algorithm such that total wavelength channel cost is minimized is NP-hard as it can be reduced to the standard Steiner tree problem when the sparse-splitting constraint is relaxed.

This work presents a novel all-optical multicast tree for a given multicast session that minimizes the total number of wavelength channels needed. This tree has a single light-tree and, thus, uses a single transmitter and multiple wavelengths per session in sparse-splitting WDM networks. MC-based multicast approach is adopted, in which a light-tree is comprised of an MC tree and a set of light paths connecting MI nodes to the MC tree. In the following sections, how a minimum-cost MC tree is grown is first described. The procedure is then extended to a complete light-tree with both MC and MI nodes using two algorithms.
For MC-based multicast schemes, the resulting light-tree consists of an $\mathrm{MC}$ tree $T_{\mathrm{MC}}$ and a set of light paths $L T$ to connect $\mathrm{MI}$ nodes with the remaining destinations to the $\mathrm{MC}$ tree. Thus, the wavelength channel cost of tree $T$ is denoted as $W C(T)(1)$.

$$
W C(T)=W C\left(T_{M C}\right)+\sum_{L_{T}^{i} \in L_{T}} W C\left(L_{T}^{i}\right)
$$

The primary design goal was to develop an efficient algorithm that minimizes $W C\left(T_{M C}\right)$. This problem is, however, is NP-hard as it can be reduced to the standard Steiner tree problem when the sparse-splitting constraint is relaxed. The standard approach in existing work (e.g., [2-5]) for constructing a light-tree is to combine an existing IP multicast routing algorithm with rerouting procedures. Here we aim at eliminating the extra rerouting for light-tree construction. In the proposed solution, an auxiliary MC network $M_{G}$ is first built from the original network $G$, and an existing Steiner tree heuristic is then applied to $M_{G}$ to obtain a minimum-cost MC tree. The $M C$ network $M_{G}$ in $G(E, V)$ is a network with the following characteristics.

- All MC nodes in $G(E, V)$.

- Two MC nodes in $M_{G}$ are connected if there is a path between these two MC nodes in $G(E, V)$.

Link cost from node $i$ to node $j$ in $M_{G}$ is equal to the cost of the minimum-weight path from node $i$ to node $j$ in $G$.

\section{The Proposed Algorithms}

The proposed algorithm includes three phases. The first phase is the MC node placement, in which the maximum path count first (MPCF) algorithm is used to obtain candidates for MC nodes. The second phase is a multicast routing with MC-based schemes, in which PMC and PSP algorithms construct an MC-based tree. The third phase utilizes a maintainability mechanism that works after MC node placement and MC-based tree construction.

\subsection{Cost Function}

The conversion times of MCs and MIs are ignored and the cost function of each link is assumed the transmission delay in this paper. The detail of the proposed algorithm is described in detail as follows. The corresponding equation [7] is (2):

$$
\tau=\rho+\sigma+0.25 \times \omega
$$


Where $\tau$ is the transmission delay, $\rho$ is the propagation delay, $\sigma$ is the node processing time, and $\omega$ is the processing delay of wavelength converter.

\subsection{Nodes Placement}

An effective means of selecting a candidate MC node, namely, MPCF, is proposed and described as follows.

1. First, find the shortest paths with minimum transmission delay for all pairs of nodes in a given network.

2. For each network node, the number of the shortest paths with minimum transmission delay passing through the node is assigned as the count of the node.

3. Nodes are then sorted in descending order according to their path counts.

4. The first $N$ nodes are selected as the MC node.

The proposed algorithm is analyzed by simulating the NSFNET and the number beside each link specifies the transmission delay with the link [8]. The candidate nodes for MC in order are $\{9,4,8,5,2,7,6$, 13, 11, 12, 14, 10, 3, 1\} (Figures 1).

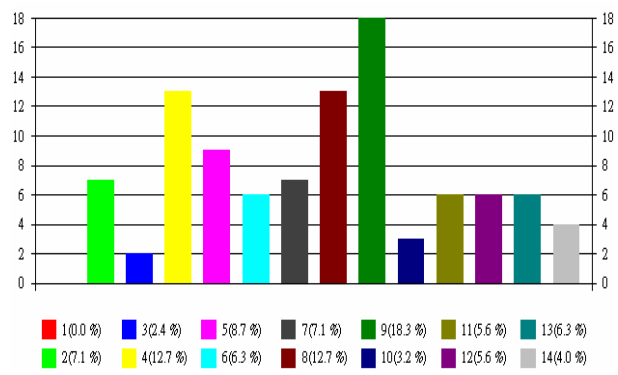

Fig.1: Assessment of MC Placement in NSFNET

\subsection{Multicast Routing}

In [6], we have proved that finding an MC-tree with minimum cost is equivalent to finding a Steiner tree in the corresponding MC network. We can apply any existing Steiner Tree heuristic for a multicast session in the MC network to obtain the minimum-cost MC-Tree. The MC-Tree is then transformed back to the original network to yield the resulting light-tree. We call such approach as Pre-computing Minimum Cost Tree (PMC). Any existing shortest-path heuristic (Dijstra's algorithm [9]) for a multicast session in the MC network can be applied to obtain the shortest-path MC-tree. Such approach is called the pre-computing shortest path tree (PSP).

The example provided in Figures 2 demonstrates how PMC and PSP work; the circles denote MI nodes and the squares represent MC nodes placed in $\{4,8,9\}$.
Node 10 is the source and the other shaded nodes are destinations. Figures 2(a) shows the resulting MC-tree with a cost of 16.8 generated by PMC. Figures 2(b) shows the resulting MC-tree with a cost of 20.3 produced by PSP.

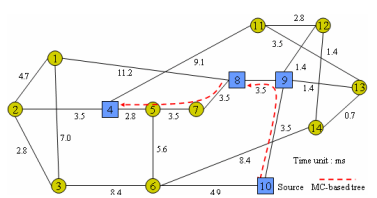

(a) $\mathrm{PMC}$

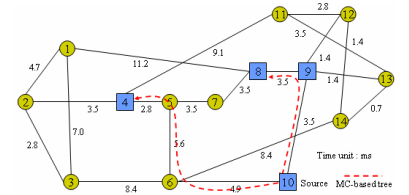

(b) PSP
Fig. 2: Constructing MC-tree in NSFNET

\section{Simulation}

\subsection{Simulation Environment}

1. Performance of the proposed algorithm is analyzed by simulating a 14-node NSFNET and 28-node USANET [7-8].

2. Source node $S$ and destination nodes $D$ are randomly selected. Each multicast member ratio (10-90\%) runs for 50 sessions.

3. Each MC node in the network is assumed equipped with both splitting and wavelength conversion capabilities.

4. For sparse light splitter, the placement algorithm proposed in MPCF and high-performance switching and routing (HPSR) [10].

5. Compare the performance of PMC and PSP with the RTS algorithm in terms of the average number of wavelengths needed average call blocking probability and mean maximum transmission delay.

\subsection{Performance Evaluation}

The system performance of the proposed algorithm is compared with the RTS algorithm for average number of wavelengths, average call blocking probability and maximum delay. Figures 3 plot the average numbers of wavelength are needed vs. the multicast member ratio with different $\mathrm{MC}$ ratio of $10 \%$ and $40 \%$ in NSFNET.

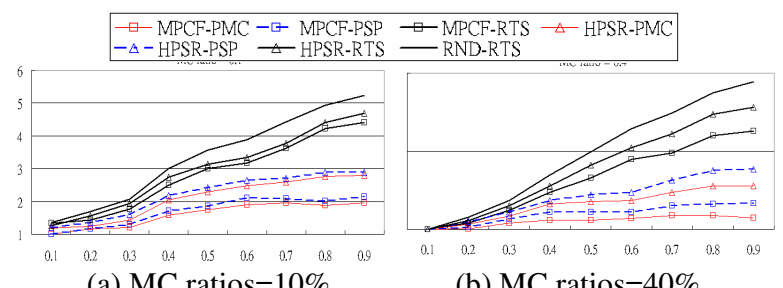

(a) $\mathrm{MC}$ ratios $=10 \%$

(b) $\mathrm{MC}$ ratios $=40 \%$

Fig. 3: Number of Wavelengths Needed vs. Multicast Member Ratio 
Clearly, the average number of wavelengths obtained using PMC and PSP is lower than that obtained using RTS algorithms. Based on figures, both algorithms reduced the number of wavelengths needed obtained by about $30-50 \%$ for $\mathrm{MC}$ ratio $=10 \%$ and $35-65 \%$ for $\mathrm{MC}$ ratio $=40 \%$. This is due to the RTS method connects directly to a source node and increases wavelength demand on the optical fiber in the multicast session.

Figures 4 plot blocking probability vs. multicast member ratio with $\mathrm{MC}$ ratios of $10 \%$ and $40 \%$ in NSFNET. As in the comparison based on number of wavelengths, the blocking probability obtained using PMC and PSP is superior to that obtained using the RTS algorithm. For all-optical networks, both algorithms reduced blocking probability obtained by about $25-50 \%$ for $\mathrm{MC}$ ratio=10\% and $30-65 \%$ for MC ratio $=40 \%$.

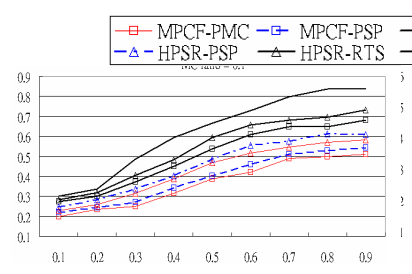

(a) $\mathrm{MC}$ ratios $=10 \%$

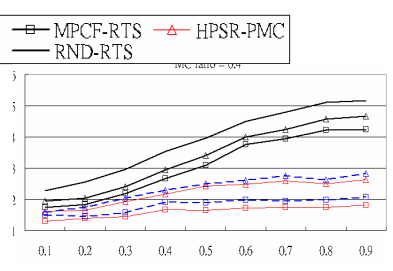

(b) $\mathrm{MC}$ ratios $=40 \%$
Fig. 4: Blocking Probability vs. Multicast Member Ratio

Figures 5 plot the mean maximum transmission delay in NSFNET. According to these figures, the RTS algorithm works better than the PMC and PSP algorithms in the multicast session. The superior performance of the RTS algorithm is due to its use of the shortest path method in constructing a multicast light tree. Also this method will utilize less transmission delay.

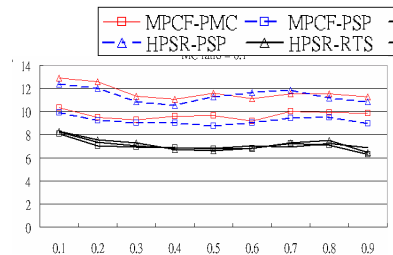

(a) $\mathrm{MC}$ ratios $=10 \%$

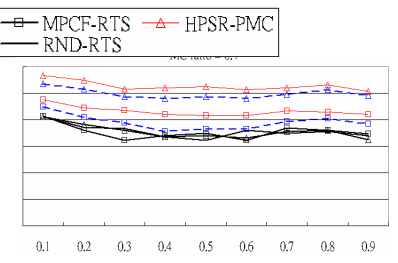

(b) $\mathrm{MC}$ ratios $=40 \%$
Fig. 5: Mean Maximum Transmission Delay (ms) vs. Multicast Member Ratio

\section{Conclusions}

This work presented two novel mechanisms for constructing an MC-based tree with minimum number of wavelengths and low blocking probability for a multicast session in optical networks with sparse light splitting. These mechanisms eliminate the requirement of rerouting. Consequently, two algorithms were developed for constructing a minimum-cost MC-tree and a shortest-path MC-tree. Simulations were utilized to compare the proposed mechanisms with RTS. Simulation results show that PMC and PSP used fewer wavelengths and had the better blocking probability than the RTS algorithm. Both algorithms reduce the number of wavelengths need by about $30-65 \%$ blocking probability by about $25-65 \%$. Both PMC and PSP have longer transmission delay.

\section{References}

[1]. L.H. Sahasrabuddhe and B. Mukherjee, "Light-trees: Optical Multicasting for Improved Performance in Wavelength-Routed Networks," IEEE Communications Magazine, vol. 37, no. 2, pp. 67-73, Feb. 1999.

[2]. X. Zhang, J.Y. Wei and C. Qiao, "Constrained Multicast Routing in WDM Networks with Sparse Light Splitting," Journal of Lightwave technology, vol. 18, Issue:12, pp. 1917-1927, Dec 2000.

[3]. S. Yan, M. Ali and J. Deogun, "Route Optimization of Multicast Sessions in Sparse Light-Splitting Optical Networks," IEEE GLOBECOM, vol. 4, 2001, pp. 2134-2138.

[4]. W.Y. Tseng and S.Y. Kuo, "All-Optical Multicasting on Wavelength-Routed WDM Networks with Partial Replication," IEEE ICC '01, Helsinki, Finland, pp. 813-818, Jun 2001.

[5]. N. Sreenath, "Virtual Source based Multicast Routing in WDM Networks with Sparse-Light Splitting, " IEEE High Performance Switching and Routing, pp. 141-145, May 2001.

[6]. C.Y. Hsieh, "All Optical Multicast in Dense Wavelength Division Multiplexing (DWDM) Networks," Master Thesis, National Taiwan University, June 2002.

[7]. I.S. Hwang, I.F. Huang and C.C. Chien, "A Novel Dynamic Fault Restoration Mechanism using Multiple Ring Approach in WDM Mesh Network," Photonic Network Communications, vol. 10, no. 1, pp. 87-105, July 2005.

[8]. S. Arakawa and M. Murata, "Lightpath management of logical topology with incremental traffic changes for reliable IP over WDM networks," Optical Networks Magazine, pp. 68-76, May/June 2002.

[9]. E.W. Dijkstra, "A note on two problems in connection with graph," Numerical Mathematics, vol. 1, pp. 269-271, Oct 1959.

[10]. V. Tamarapalli and S.H. Srinivasan, "Normalized cuts for wavelength converter placement," 2004 Workshop on High Performance Switching and Routing (HPSR), pp. 292-296, 2004. 